Rev. SINAPSIS, Vol. 4, No 1, Junio 2014

ISSN $1390-7832$

\title{
Análisis de la competencia comunicativa y su vínculo con la formación para los negocios desde la enseñanza-aprendizaje del inglés con fines específicos
}

\section{Las competencias comunicativas en la enseñanza del inglés}

Maritza Sandra Pibaque Pionce. MSc. ${ }^{(1)}$

Ned Quevedo Arnaiz. Dr. C. (2)

Silvia Colunga Santos. Dr. C. ${ }^{(3)}$

${ }^{(1)}$ Universidad Estatal del Sur de Manabí, Ecuador

${ }^{(2)}$ Universidad de Camaguey- Ignacio Agramonte Loynaz, Cuba

${ }^{(3)}$ Universidad de Camaguey- Ignacio Agramonte Loynaz, Cuba

Contacto: m1_aritza@hotmail.com

Receptado: 12 / 03 / 2014

Aceptado: 27/ $05 / 2014$

\section{Resumen}

La educación para el desarrollo hace posible que toda persona adquiera los valores, capacidades, habilidades y conocimiento necesarios para contribuir a la construcción de una sociedad, a partir del análisis efectuado se asume como problema: insuficiencias en la comunicación oral en idioma inglés, de los estudiantes de carreras universitarias relacionadas con los negocios internacionales, con el objetivo elaborar una estrategia didáctica para favorecer la formación y desarrollo de la competencia linguo -cultural, desde la enseñanza-aprendizaje del Inglés con fines específicos, Su objeto, el proceso de enseñanza aprendizaje del Inglés con fines específicos en las carreras universitarias relacionadas con los negocios internacionales, y el campo, la formación y desarrollo de la competencia linguo-cultural. Es labor primordial de los educadores brindar y fortalecer las herramientas necesarias para que el estudiante sea capaz de desarrollar una adecuada comunicación en las diversas situaciones y escenarios de la vida, esencialmente en su labor como profesional en negocios, para poder establecer una adecuada comunicación.

Palabras claves: enseñanza de lenguas, competencia comunicativa, desempeño comunicativo, comunicación, profesional en negocios, formación, negocios internacionales, inglés con fines específicos. 
Rev. SINAPSIS, Vol. 4, No 1, Junio 2014

ISSN $1390-7832$

\title{
Analysis of communicative competence and its link with the business training from the teaching and learning of English for Specific Purposes
}

\begin{abstract}
The development education enables a person to acquire the values, skills, abilities and knowledge to contribute to building a society from the preceding analysis it is assumed as a problem: inadequacies in oral communication in English, the university students of careers related to international business, with the aim to develop a teaching strategy to promote the formation and development of the lingual -cultural competence, from the english teaching learning for specific purposes, Its object, the english teaching learning for specific purposes in university careers related to international business and country, the formation and development of the lingual - cultural competence. Primary task is to provide educators and strengthen necessary for the student to be able to develop adequate communication in various situations and stages of life, essentially in its work as a professional business, to establish proper communication tools.
\end{abstract}

Keywords: language teaching, communicative competence, communicative, performance, communication, business professional, formation, English with specific purposes.

\section{Introducción}

En este artículo se valora la competencia comunicativa y su vínculo con la formación para los negocios, su evolución, la competencia comunicativa y el proceso de enseñanza aprendizaje del idioma inglés en la universidad, el inglés y los negocios y la formación para los negocios desde el inglés con fines específicos. Del mismo modo se develan los resultados del diagnóstico inicial de la competencia comunicativa y su vínculo con la formación para los negocios de estudiantes de Quinto Semestre de la Carrera de Comercio Exterior de la Universidad Estatal del Sur de Manabí (UNESUM) Jipijapa - Ecuador, en base a los cambios que los sistemas educativos están experimentando tanto en su estructura, como en su finalidades pedagógicas, dirigidas principalmente a desarrollar competencias en los estudiantes que les permitan construir conscientemente conocimiento, aprender a lo largo de la vida y afrontar con ciertas garantías de éxito futuros desafíos de aprendizaje. (Hernández, et al 2010) 
Rev. SINAPSIS, Vol. 4, No 1, Junio 2014

Investigaciones realizadas sobre el tema, se ha podido comprobar que el inglés es una forma viable de transmisión de cultura general para la profesión de los estudiantes. Ha sido valorada como cultura que hay que tomar en cuenta para el comportamiento humano en diferentes contextos interculturales, unos como competencias básicas que han sido adoptados por los sistemas educativos de los países con inmigrantes (Alsina , 2009; Rodríguez, 2010) y otros como competencias a desarrollar en currículos de carreras profesionales como profesores, enfermeras y otros. Santamaría (2008) y las tesis doctorales en el área de la ingeniería comercial se han dirigido a la consideración del desarrollo de la lengua materna en la competencia cultural para los negocios por lo que analiza los materiales para trabajarla Riutort ( 2010) y el vínculo universidad-empresa relacionado con el nivel de competitividad de los países Hernández (2008). No se ha analizado aún la relación del desarrollo de la competencia linguo cultural desde el inglés para carreras relacionadas con los negocios.

\section{Métodos}

Para realizar la competencia comunicativa y su vínculo con la formación para los negocios, se aplicó el método deductivo a partir de situaciones generales explicadas por un marco teórico general aplicado a competencia comunicativa, inductivo enfocado de manera particular generalizándolas para poder esclarecer la competencia comunicativa y el proceso de enseñanza aprendizaje del inglés en la universidad, analítico basado en el análisis de datos obtenidos, comparar y hacer analogías; y sintético para realizar el análisis de la competencia comunicativa; hermenéutico - dialéctico: para interpretar el significado de los sustentos teóricos que confluyen en la competencia comunicativa y su vínculo con la formación para los negocios en estudiantes de carreras de negocios internacionales.

\section{Resultados}

A pesar de que el enfoque comunicativo, concepción moderna, que se utiliza en los profesionales de comercio exterior en el país, especialmente en la universidad Estatal del Sur de Manabí para la enseñanza del inglés; tiene como principio lograr la competencia comunicativa en los futuros profesionales a través de la comprensión, análisis y construcción de textos de forma coherente, no siempre se logra este objetivo primordial.

Las competencias comunicativas en la enseñanza del inglés www.itsup.edu.ec/myjournal 
Rev. SINAPSIS, Vol. 4, No 1, Junio 2014

ISSN $1390-7832$

Primeramente se hace necesario comentar que en la propia enseñanza de la lengua extranjera ha imperado el formalismo, así como el divorcio entre el análisis reflexivo y el empleo práctico de la misma. Se ha hecho patente la insuficiente planificación y orientación hacia objetivos determinados, la tendencia al repetitismo y a la ejercitación de las habilidades comunicativas, desvinculadas de la práctica social.

El desarrollo de las habilidades comunicativas, a pesar de ser en esencia un proceso activo, no ha escapado al formalismo. Esto ocurre sobre todo cuando se ha querido desarrollar dichas habilidades en situaciones desligadas de las necesidades práctico comunicativas, del estudiante, sin analizar que las actividades comunicativas que son el centro del esqueleto u organización conceptual de la enseñanza comunicativa del lenguaje, involucran el aprendizaje en comunicación auténtica y que además esto puede tener lugar en un contexto de interacción entre dos personas en una de las infinitas situaciones de la vida diaria; pero que también puede ser organizada para aprender fines, propósitos en el contexto de la clase.

\section{Desarrollo evolutivo del término competencia comunicativa}

Hoy en día, en la consolidación del enfoque de competencias en la educación no solo han influido los desarrollos disciplinares, sino también el momento histórico y la economía. En lo social, se tienen las progresivas presiones del sistema para que la educación forme para la vida y para el trabajo con calidad, y trascienda el énfasis en lo teórico y la transmisión de la información, porque en la Sociedad del Conocimiento, lo más importante no es tener conocimiento sino saberlos buscar, procesar, analizar y aplicar con idoneidad. Respecto a lo económico, se ha creado la demanda de las empresas a las instituciones educativas para que formen profesionales idóneos, de tal manera que esto les permita competir con otras empresas nacionales e internacionales para mantenerse y crecer. En síntesis, el auge de las competencias en la educación se corresponde con una mayor implicación de la sociedad en la educación, la cultura de la calidad, la globalización y la competitividad empresarial.

Esta construcción histórica del concepto de competencias en torno a múltiples referentes disciplinares y demandas socioeconómicas es lo que explica la diversidad de definiciones y 
Rev. SINAPSIS, Vol. 4, No 1, Junio 2014

metodologías para llevar a la práctica el enfoque de competencias. Sin embargo, en esta característica, que se ha visto como una falencia sustancial de las competencias, es donde precisamente reside la mayor integridad del enfoque de competencias, en tanto su naturaleza tiene una visión multidisciplinar y esto hace que este enfoque pueda alimentarse de diferentes contribuciones. Así mismo, esta naturaleza multidisciplinar de las competencias es lo que permite que sean asumidas como un lenguaje común para referirse al talento humano tanto en las organizaciones educativas, como en las organizaciones sociales y en las organizaciones empresariales, facilitando esto la articulación de la educación con lo social y lo económico.

El término competencia fue en primera instancia usado por Noam Chomsky, lingüista famoso, innovador e influyente del siglo XX, conocido también por sus escritos sobre política, historia y economía, porque 'Desarrollar' una lengua implica descubrir las unidades, estructuras y reglas formales que articulan la lengua del medio, o, adquiere una competencia lingüística, entendida como un sistema de conocimiento interiorizado, atendiendo a un conjunto de reglas que ofrezca a las frases gramaticales modelos de lengua, es decir, una gramática dirigida no-solo, a describir los enunciados sino a explicar cómo han sido producidos. (Chomsky,1989)

Hymes (1996), desarrolla un concepto de competencia comunicativa que supera la teoría chomskiana en cuanto a la competencia lingüística, propone el concepto de «competencia comunicativa», que incluye las reglas de uso a las que hace referencia. Incluye el significado referencial y social del lenguaje, y no solo se refiere a la estructura de las oraciones, sino también a si estas son apropiadas o no en el contexto, y presenta cuatro dimensiones: el grado en que algo resulta formalmente posible (gramaticalidad), el grado en que algo resulta factible, el grado en que algo resulta apropiado y el grado en que algo se da en la realidad. También afirma que la competencia es el conocimiento subyacente general y la habilidad para el uso de la lengua que posee el hablante-oyente, considerando factores que intervienen en la comunicación cuando usan la lengua. Estos incluyen las características de los interlocutores o las relaciones que unen al interlocutor.

Tomando como referente lo antes mencionado se analiza que, para Chomsky, la competencia es el conocimiento gramatical como un arraigado estado mental por debajo del nivel de lengua. No es una habilidad para hacer nada. Ni siquiera es la habilidad para formar o comprender oraciones, 
Rev. SINAPSIS, Vol. 4, Nº 1, Junio 2014

porque el conocimiento puede existir sin que sea accesible (...). Para Hymes, la competencia es la habilidad para hacer algo: Usar la lengua. Para él, el conocimiento gramatical es un recurso, no una configuración cognitiva abstracta existente por propio derecho como una estructura mental.

Las apreciaciones sobre competencia comunicativa por Savignon (1983) plantean "eficiencia funcional en el uso del lenguaje; la expresión, interpretación y negociación de significado que involucra la interacción de dos o más personas que pertenecen a la misma o a diferentes comunidades lingüísticas, o entre una persona y un texto oral o escrito". Esta definición contrasta por su riqueza en ese momento con las definiciones tradicionales de competencia gramatical como conocimiento de las estructuras gramaticales de la lengua. Se destaca en ella la referencia a la comunicación sujeto-texto oral o escrito, que contrasta con posiciones reduccionistas del propio enfoque que limitan su aplicación a las habilidades de expresión oral y escrita.

Stern (1979), en el desarrollo de la competencia comunicativa en hablantes de una segunda lengua y divide su contenido en cuatro bloques, a los que denomina: Language Syllabus, Culture Syllabus, Communicative Activity Syllabus y General Language Education Syllabus, todos ellos orientados a lograr en los estudiantes las destrezas, conocimientos, tanto de la lengua como de la cultura-, motivaciones y compromisos o implicación personal y las técnicas de adquisición de una lengua y su análisis para desarrollar actitudes positivas hacia el aprendizaje de idiomas, en sentido general.

Byram (2007) potencia, dentro del concepto de competencia comunicativa, la competencia sociocultural y, dentro de esta, propone el término 'hablante intercultural' que no es más que "los interlocutores involucrados en la comunicación e interacción cultural". Se establece la diferencia entre competencia intercultural y la competencia comunicativa intercultural, donde todo estudiante necesita y debe abordar el descubrimiento de una cultura extranjera con actitudes y saberes (saber ser, saber hacer, saber aprender y los saberes -lingüístico y cultural)

Desde el punto de vista psicológico, Zaldívar (1998) considera, como elementos inherentes a la competencia comunicativa, los conocimientos, habilidades, actitudes, valores y comportamientos necesarios para establecer una comunicación y relaciones interpersonales positivas. 
Rev. SINAPSIS, Vol. 4, No 1, Junio 2014

ISSN $1390-7832$

Tres componentes fundamentales dentro del concepto de competencia comunicativa y cada uno de ellos, a su vez, consta de tres elementos: competencia existencial o saber ser, conocimientos declarativos o saberes y destrezas o saber hacer. Trujillo (2001) plantea que en los componentes se encuentran el socio-lingüístico, el lingüístico y el pragmático.

Como se puede apreciar, el concepto de competencia comunicativa se ha redimensionado y enriquecido en su desarrollo al introducir otras competencias como la intercultural, socio-cultural, de aprendizaje, etc. Prueba de esto es, la introducción de la competencia cognitiva que, de acuerdo a lo planteado por Roméu ( 2007) no es más que "la habilidad de construir y reconstruir conocimientos a través de la lengua". Y la competencia afectiva que se define, de acuerdo a lo planteado por Vilá (2003) como "la habilidad para reconocer, expresar y canalizar la vida emocional, donde adquiere especial importancia el equilibrio personal, la autoestima y la empatía, así como la meta-afectividad o capacidad del sujeto para conocer y gobernar los sentimientos que provocan los fenómenos afectivos".

De acuerdo con Más (2004) la competencia comunicativa, como proceso, debe ser formada en el aula, lo cual significa abordar elementos de las dos esferas básicas de la personalidad: la motivacional-afectiva y la cognitiva-instrumental.

Para Beltrán (2007) la competencia comunicativa se asocia al estudio de una lengua y el modo de alcanzarla es a través del dominio de los diferentes saberes (saber lingüístico, saber hacer y saber ser).

Pulido (2008, p.1) define la competencia comunicativa integral como "la habilidad del que aprende la lengua para expresar, interpretar y negociar significados socio-culturales en la interacción entre dos o más personas o entre una persona y un texto oral o escrito, de forma tal que el proceso de comunicación sea eficiente y esté matizado por modos de actuación apropiados".

Como se ha podido apreciar a lo largo de este análisis, como bien expresó en sentido general, los cambios de nombres no harán la diferencia. La extensión del conocimiento, sí, porque alcanzar la 
Rev. SINAPSIS, Vol. 4, No 1, Junio 2014

competencia comunicativa implica la apropiación de conocimientos, habilidades y valores en el proceso de enseñanza aprendizaje del idioma inglés.

Lo anteriormente expuesto hace posible arribar a regularidades que se consideran válidas dentro del concepto de competencia comunicativa:

a. La competencia comunicativa debe su surgimiento y posterior desarrollo y evolución a diversas ramas de la ciencia como la Psicología, Pedagogía, Sociología y Linguística.

b. La estructura interna o elementos constitutivos de la competencia comunicativa agrupa tanto factores verbales como no verbales, donde el análisis de la cultura y la sociedad de la lengua meta juegan un papel fundamental.

c. Es el aula el lugar adecuado para formar y fomentar comunicadores competentes.

Se observa, además, que aparejado a la evolución de dicho concepto surge, a principios del siglo XX, a través de la obra Threshold Level of the Council of Europe, el enfoque comunicativo, que no es más que el supuesto teórico que sirve de base al desarrollo de la competencia comunicativa y sus aplicaciones didácticas en el aula, por medio de las cuales el estudiante podrá alcanzar dicha competencia.

Para Samerón y Rodríguez (2010): , competencia es la actuación eficiente en el desempeño de la actividad. Regula el desempeño real del sujeto en un rol, empleo o profesión". Al considerar la competencia como desempeño real, la competencia no puede reducirse a una capacidad o potencialidad. Se trata de saber actuar en determinadas situaciones y movilizar los recursos necesarios para lograr un objetivo. Este saber actuar, en los momentos actuales no solo incluye lo que realiza el estudiante en el aula, sino además la forma en que los procesos de aprendizaje continúan evolucionando fuera del ámbito educativo.

En el amplio campo investigativo se define la competencia comunicativa como el desempeño del sujeto en su actividad verbal y no verbal en situaciones reales de comunicación que involucran la interacción entre dos o más personas, o entre una persona y un texto oral o escrito, en correspondencia con un contexto social determinado. Font (2006). Lo que se debe especificar que la situación real de comunicación en el proceso de enseñanza aprendizaje del idioma extranjero es 
Rev. SINAPSIS, Vol. 4, No 1, Junio 2014

una situación simulada de lo que el estudiante puede realizar en un contexto donde esa lengua es el medio de comunicación.

Otro de los aportes se enfoca en lo contextual, basado en la psicología cultural que tiene como principal representante a Vigotsky (1985) donde el concepto de competencias es el principio de que la mente y el aprendizaje son una construcción social y requieren de la interacción con otras personas, influenciado por el mismo contexto. Tobón (2006); es decir la mente se construye en relaciones sociales y es actualizada por la cultura.

Otro perfil disciplinar sobre competencias es la psicología cognitiva, destacando la teoría de las inteligencias múltiples de Gardner $(1987,1997)$ la cual da un apoyo teórico sustancial a la comprensión de las competencias en su dimensión cognoscitiva (Tobón, 2006)

La competencia comunicativa y el proceso de enseñanza aprendizaje del inglés en la universidad Uno de los aspectos centrales del proceso enseñanza-aprendizaje en las universidades es el desarrollo de una eficaz competencia comunicativa en los estudiantes, la didáctica de la lengua debe priorizar el trabajo con este concepto y su aplicación en todos los componentes que rigen la enseñanza del idioma inglés.

Hoy más que nunca resulta imprescindible aprender el idioma inglés. Cada día se emplea más en casi todas las áreas del conocimiento y desarrollo humanos. Prácticamente puede afirmarse que se trata de la lengua del mundo actual. Es, en la era de la globalización, la gran lengua internacional, una "lingua franca" que ha trascendido en todos los países no-anglosajones, incluida España, y que afecta más o menos directamente a los diversos campos y profesiones. Su posesión ya no puede tratarse como un lujo, sino que es una necesidad evidente. Se trata de la herramienta que permite la comunicación con personas de otros países, dentro del mundo globalizado en que se vive. Es indiscutible que se ha convertido en el idioma global de comunicación por excelencia, uno de los de mayor uso en el mundo.

Por tanto, en estas dos últimas décadas se han ido produciendo importantes cambios en la teoría, la investigación y la experiencia en el aula de enseñanza de idiomas, lo que ha dado lugar a un proceso innovador, entre los que se destacan los diseños curriculares y cómo se planifican y 
Rev. SINAPSIS, Vol. 4, No 1, Junio 2014

ISSN 1390 - 7832

ponen en práctica las unidades didácticas. Son, en definitiva, nuevas representaciones de filosofías educativas, enfoques acerca de la naturaleza de la lengua y cómo puede enseñarse y aprenderse, concepciones acerca de qué tipo de interacciones deben producirse en el aula, así como filosofías y valores respecto a la sociedad y la convivencia humana las que están en juego. (Candlin, 1987)

(Breen, 1990), hace referencia a una nueva representación de cómo enseñar y aprender idiomas, un nuevo paradigma, en el que se unen teoría, investigación y práctica, centrado en el proceso.

Para la enseñanza del idioma inglés deben considerarse las cuatro dimensiones de la competencia comunicativa que en 1980 propone Canales y Swain, las cuales se relacionan con un análisis inter-disciplinario y sistemático del texto.

1. Competencia gramatical lingüística: La didáctica de la lengua debe atender al dominio de las estructuras lingüísticas del idioma de forma gradual sistémica, favoreciendo al estudiante la capacidad no solo de dominar la realidad, sino también de predicar sobre ella a partir del conocimiento previo que posee de las diferentes estructuras lingüísticas que reintegran el sistema hasta lograr la creación de un todo coherente de intención comunicativa.

2. Competencia sociolingüística: La actividad lingüística es eminentemente social y este carácter es el que establece un uso restrictivo al proceso de la comunicación, en dependencia de las situaciones comunicativas en que se desenvuelve.

3. Competencia discursiva: Dentro de los niveles constructivos del texto aparecen los sintagmas, con una función básicamente denominativa: la oración.

4. Competencia estratégica: Se relaciona con la capacidad que desarrolla el estudiante de elaborar la estrategia de comunicación para iniciar, continuar y finalizar el proceso comunicativo. El educando es capaz de cumplir una función comunicativa dada y desarrollar así, la capacidad de comprender, analizar y recrear el texto a favor del desarrollo de la competencia comunicativa.

De acuerdo con Finocchiaro (1989). la competencia comunicativa se enmarca en la habilidad de usar el sistema de la lengua de forma apropiada en cualquier circunstancia. 
Rev. SINAPSIS, Vol. 4, No 1, Junio 2014

ISSN $1390-7832$

Savignon (1983), hace referencia a la consideración de la competencia en términos de expresión, interpretación y negociación del significado incluye interacción de dos o más personas perteneciente a la misma o diferente comunidad del habla, o entre una persona y un texto escrito u oral. (Savignon, 1983).

La competencia comunicativa en el idioma inglés toma como punto de partida el Enfoque Comunicativo, dirigido al desarrollo de conocimientos, habilidades y hábitos que permiten la emisión, recepción y negociación efectiva y apropiada de los mensajes contenidos en los actos de comunicación, mediante el lenguaje verbal y no verbal, con personas anglo parlantes. En los programas de estudio se considera la primacía natural del uso oral del lenguaje sobre sus formas escritas.

El alcance de la competencia comunicativa en la enseñanza del inglés en carreras de negocios internacionales se divide por niveles: el nivel elemental, representa limitadas posibilidades de recepción y emisión de mensajes tanto por el carácter básico del conocimiento del sistema lingüístico, como por el insuficiente desarrollo de habilidades para su uso apropiado y fluido. En este nivel la competencia comunicativa de los estudiantes se sitúa en la fase de primer encuentro con un sistema lingüístico desconocido y con una comunidad y cultura de normas y costumbres diferentes a los propios.

El alumno está obligado a comparar y confrontar sus propias experiencias y convicciones con esas nuevas. La traducción desde la lengua materna y hacia ella, es un recurso natural en todo esfuerzo consciente de apropiación de otro idioma lo que puede aprovecharse cuando sea útil para señalar diferencias, equivalencias estructurales o semánticas, reconociendo que limita la fluidez en el procesamiento de los mensajes y que constituye una habilidad adicional más compleja, como un fin en sí misma.

Como etapa inicial cualquier apresuramiento por lograr niveles de expresión imposibles de alcanzar en este nivel redundará en frustraciones y pérdida de la motivación, o en el mejor de los casos, en la memorización y reproducción formal de expresiones que, aunque puedan dar la impresión de comunicación efectiva, solo son útiles para fijar patrones y vocabulario del idioma extranjero.

Las competencias comunicativas en la enseñanza del inglés www.itsup.edu.ec/myjournal 
Rev. SINAPSIS, Vol. 4, No 1, Junio 2014

ISSN $1390-7832$

En relación a avances y nuevos conocimientos en la competencia comunicativa el alumno debe utilizar de forma eficiente la lengua extranjera en situaciones comunicativas de su esfera profesional y social e incurrir en pocos errores lingüísticos. La comunicación de los estudiantes debe ser aceptable en cuanto a su efectividad y consistencia. Se producen en este nivel por parte de los estudiantes, pocas dificultades y el alumno es capaz de restablecer la comunicación si esta se interrumpe. En este nivel se comienza el Ciclo de Inglés con fines específicos, por lo que se inicia el desarrollo de hábitos y habilidades lingüístico-comunicativas propias de las situaciones de la esfera comercial., se aplicaron evaluaciones en forma oral y escrita, donde el estudiante pueda expresarse de carácter coherente e inteligible, siguiendo un proceso de aproximaciones sucesivas.

A pesar de que el enfoque comunicativo, concepción moderna, que se utiliza en las carreras universitarias para la enseñanza de lenguas extranjeras; tiene como principio lograr la competencia comunicativa como objetivo primordial y aspirar a cambios cualitativos en las estructuras y sistemas de conocimientos, que irradien también cambios cuantitativos en el proceso.

Las apreciaciones de Holder, R. (2011) sobre la enseñanza de lenguas indica que se debe instaurar definitivamente el análisis de la competencia comunicativa, de los futuros egresados, vista como el desempeño comunicativo del futuro profesional.

El proceso de formación del profesional en la actualidad tiene que garantizar que este sea dotado de todas las herramientas necesarias para su futuro desempeño laboral, ha de ser un asiduo estudioso de los cambios generados por la ciencia y la tecnología, las que le van imprimiendo a la sociedad nuevas imposiciones que él debe interpretar, explicar en función de buscar soluciones a las nuevas exigencias socioculturales.

Esta realidad demanda de las universidades en el contexto actual un cambio en lo referido a la formación del profesional, como refiere Horruitineir (2009): El paradigma está en brindar a la sociedad un profesional formado de manera íntegra, profesionalmente competente, con preparación científica para aceptar los retos de la sociedad moderna y con un amplio desarrollo 
Rev. SINAPSIS, Vol. 4, No 1, Junio 2014

ISSN 1390 - 7832

humanístico para vivir en la sociedad de esta época y servirla con sencillez y modestia, con los valores como pilar fundamental de su formación.

El inglés y los negocios

La correcta formación incluye el entrenamiento en la lengua inglesa, desde el nivel básico hasta superior, donde se debe desarrollar cada vez más la capacidad de pensar, razonar y actuar creativamente, ampliar el uso de los métodos activos de la enseñanza para las habilidades prácticas y la solidez de los conocimientos de los egresados. Así mismo, la formación debe ser capaz de dotar a los estudiantes del idioma de las herramientas necesarias para abordar situaciones reales de negociación, presentación de empresas y productos, etc. en función de las necesidades de negocios.

En la actualidad la necesidad de dominar el inglés es cada vez de mayor importancia, además de elevar la eficiencia profesional, desarrolla relaciones de amistad y solidaridad, contribuye a la comprensión intercultural y a la formación de valores éticos y estéticos y aporta una de las soluciones para la necesidad de comunicarse en inglés.

Se infiere entonces, que a través del trabajo con contenidos de negocios internacionales en inglés, los profesionales en formación de las especialidades de Comercio, Turismo, Economía, Informática, entre otras, comienzan a utilizar el inglés para propósitos específicos; en actividades relacionadas con la educación en el campo laboral tales como: discusiones de negocios, conferencias comerciales, entrevistas a comerciantes, reportes de negocios, paneles y mesas redondas. Entre otras se pueden relacionar las búsquedas bibliográficas y la consulta bibliográfica especializada.

Toda esta preparación, abre las puertas al negocio internacional ecuatoriano en países de habla inglesa; sin embargo, no se percibe la necesidad de integración de la formación especializada con la formación general, desatendiéndose en este sentido la formación idiomática en idioma inglés, lo cual ha traído como consecuencia que muchos de los estudiantes de negocios internacionales no desarrollen su competencia comunicativa en idioma inglés de manera apropiada (Kippel,1984) 
Rev. SINAPSIS, Vol. 4, No 1, Junio 2014

ISSN $1390-7832$

Considerándose que el lenguaje humano constituye, por su propia naturaleza, una forma de expresión del pensamiento y un instrumento insustituible para la comunicación entre los seres vivos, la comunicación constituye el único medio de compartir con los demás los conocimientos, las informaciones, las experiencias y las vivencias. Es el soporte que crea, desarrolla y consolida las relaciones sociales.

Específicamente la enseñanza del inglés debe instaurar definitivamente el análisis de la competencia comunicativa, de los futuros profesionales, vista como el desempeño comunicativo de los estudiantes al integrar las cuatro habilidades del lenguaje: hablar, escuchar, leer y escribir.

Dentro de las características fundamentales de este enfoque comunicativo se destacan las siguientes:

La lengua es un sistema para la expresión de significados.

La función primaria de la lengua es la interacción y la comunicación.

La estructura de la lengua refleja sus usos funcionales y comunicativos.

\section{Discusión}

Uno de los problemas atendidos con prioridad en Ecuador, es el relacionado con la formación de profesionales, capaces de llevar los niveles de inglés a índices que hoy compiten con los resultados de muchas naciones desarrolladas, donde la enseñanza de tipo comunicativo es aquella que se marca como objetivo el desarrollo de la competencia comunicativa. La esencia de este enfoque se encuentra en el enriquecimiento de los objetivos de aprendizaje: no se trata únicamente de que los estudiantes adquieran un determinado sistema lingüístico, sino de que sean capaces de utilizarlo para comunicarse de forma adecuada y efectiva.

Se coincide con Horruitineir, S., en ofrecer a la sociedad un profesional formado de íntegramente, profesionalmente competente, con preparación científica, con un vasto desarrollo humanístico para vivir en la sociedad de esta época.

Las competencias comunicativas en la enseñanza del inglés www.itsup.edu.ec/myjournal 
Rev. SINAPSIS, Vol. 4, No 1, Junio 2014

Metodológicamente los estudiantes deben leer intensivamente para reforzar las habilidades cognitivas fundamentales (comparación, definición, argumentación, caracterización) con contenidos de IFE, lo cual sería un reto desafiante y motivador de la creatividad, vinculando el inglés y los negocios.

\section{Conclusiones}

Por su importancia, no solo en la enseñanza de lenguas, se hace necesario su estudio y profundización en aras de perfeccionar y enriquecer los contenidos y programas que conforman las carreras relacionadas a los negocios internacionales y, de este modo, dar un paso de avance en los esfuerzos para favorecer la formación y desarrollo de la competencia linguo -cultural, desde la enseñanza-aprendizaje del Inglés con fines específicos

El análisis de las insuficiencias en la comunicación oral en idioma inglés, de los estudiantes de carreras universitarias relacionadas con los negocios internacionales se constituye, en el eje sobre el que se articula la actuación de los docentes, en búsqueda de alternativas metodológicas hacia cambios cualitativos en las estructuras y sistemas de conocimientos, que irradien también cambios cuantitativos en el proceso.

\section{Bibliografía}

1. Beltrán, F. (2010). Desarrollo de la competencia comunicativa. Rev Edu Mex.

2. Michael P. (1990) Paradigmas actuales en el diseño de programas de lenguas. Centro Virtual Cervantes.

3. Byram M \& Zárate, G. (2007). La competencia socio-cultural en el aprendizaje de lenguas extranjeras.

4. Candlin, C. (1987): Towards task-based language learning, en Language Learning Tasks. C. Candlin and D. Murphy (eds.). Englewood Cliffs, N.J.: Prentice Hall. CANDLIN, C. AND D. MURPHY (eds.) (1987): Language Learning Tasks. Englewood Cliffs, N.J.: Prentice Hall.

5. Canale, M. (1995): Londres: Longman.

6. CHOMSKY, N. (1965) Aspects of the Theory of Syntaxt. Cambridge, Mass, MIT Press.

7. Finocchiaro, M. 1989. The Functional Notional Approach, From Theory to Practice. Edición Revolucionaria.

8. Font, Sergio, 2006. Metodología para la asignatura Inglés en la Secundaria Básica desde una perspectiva problemática del Enfoque Comunicativo. I.S.P. E.J. Varona, La Habana

9. Gardner, H. (1997) Las estructuras de la mente. La teoría de las inteligencias múltiples: México.

10. Hernández D. (2007) Competencias comunicativas. UNAD. Bogotá - Colombia. 
Rev. SINAPSIS, Vol. 4, Nº 1, Junio 2014

11. Hernández Pina, F., Rosario, P. \& Cuesta, J.D. (2010). A self-regulated learning intervention programme: Impact on university students. Revista de Educación, (353), 317-318

12. Holder, Roberto Erasm o. (2011) La competencia comunicativa y su relación con la enseñanza del idioma inglés. Grupo Eumed.net (Universidad de Málaga), ISSN 1989-4155.

13. Horruitiner, Silva P. (2009). La universidad Cubana: El modelo de formación. La Habana. Editorial Universitaria.

14. Hymes, D. H. (1971). «Acerca de la competencia comunicativa». En Llobera et al. (1995). Competencia comunicativa. Documentos básicos en la enseñanza de lenguas extranjeras. Madrid: Edelsa.

15. Hymes, D. (1996) Acerca de la competencia comunicativa. Forma y Función, 9 Departamento de Lingüística, Universidad Nacional de Bogotá.

16. Klippel, F. 1984. Talking. Communicative Fluency Activities for language teaching. New York. CUP.

17. Parra J, Más P. (2004) La competencia comunicativa profesional pedagógica: una aproximación a su definición.

18. Pulido A, Pérez V. (2008) Hacia un concepto de competencia comunicativa integral.

19. Roméu Escobar, Angelina (2007). El enfoque cognitivo, comunicativo y sociocultural en la enseñanza de la lengua y la literatura. La Habana. Editorial Pueblo y Educación.

20. Salmerón, Gutiérrez-Braojos, Rodríguez-Fernandez, \& Salmeron-Vilchez, 2010. Influencia del aprendizaje cooperativo en el desarrollo de la competencia para aprender a aprender en la infancia. Revista Española de Orientación y Psicopedagogía. VOL, 21,308-319.

21. Savignon Sandra: Communicative Competence. Theory and Classroom Practice. Texts and contexts in second language learning. - Reading, Mass. : Addison-Wesley, 1983.p.98 (En Soporte Digital)

22. Stern, H. H. (1979): "Language learning on the spot: some thoughts on linguistic preparation for bilingual student exchanges". Toronto: The Ontario. Instituto for Studies in Education. Mimeo.

23. Tobón, S. (2006) Las competencias en la educación superior: políticas de calidad. Bogtá: ECOE

24. Tobón, S. (2006) Formación basada en competencias.: Pensamiento complejo, diseño curricular y didáctica: Bogotá: ECOE.

25. Trujillo F. (2001) Objetivos en la enseñanza de lenguas extranjeras: De la competencia linguiística a la competencia intercultural. Didáctica de la Lengua y la Literatura Facultad de Educación y Humanidades de Ceuta Universidad de Granada.

26. Vilá, R. (2003). La competencia comunicativa intercultural en Educación Secundaria Obligatoria: Escala de sensibilidad intercultural. En E. Soriano (Ed.), Perspectivas teórico prácticas en educación intercultural. Almería: Publicaciones de la Universidad de Almería.

27. Zaldívar F. (1998) Competencia comunicativa y relaciones interpersonales. 
Rev. SINAPSIS, Vol. 4, Nº 1, Junio 2014 